\title{
Fístula parotídea após acesso retromandibular: relato de caso
}

\author{
Parotid fistula after retromandibular access: case report
}

Fístula parótida después del acceso retromandibular: reporte de un caso

\author{
Felipe Aurélio GUERRA ${ }^{\mathbf{1}}$ \\ Eleonor Álvaro GARBIN JÚNIOR ${ }^{2}$ \\ Geraldo Luiz GRIZA ${ }^{2}$ \\ Natasha Magro ÉRNICA ${ }^{2}$ \\ Ricardo Augusto CONCI $^{2}$
}

${ }^{1}$ Residente em CTBMF, Universidade Estadual do Oeste do Paraná UNIOESTE 85819-110, Cascavel-PR, Brasil

${ }^{2}$ Professor(a) Doutor(a), Universidade Estadual do Oeste do Paraná UNIOESTE 85819-110, Cascavel-PR, Brasil

\begin{abstract}
Resumo
Introdução: O tratamento de injúrias ao esqueleto facial exige por vezes acessos extraorais para uma melhor visualização, redução e fixação das fraturas. Em fraturas condilares o acesso retromandibular é largamente utilizado na prática clínica, apresentando previsibilidade e campo operatório adequado, todavia pode gerar complicações como parestesia, paresia/paralisia, sialocele e fístulas parotídeas. Objetivo: relatar um caso clínico de fístula parotídea após acesso retromandibular bem como a conduta aplicada no tratamento. Resultados: É consenso na literatura que injúrias agudas à glândula parótida devem ser prontamente corrigidas, no entanto danos pós-operatórios crônicos apresentam uma vasta gama de tratamentos. Mesmo condutas extremamente conservadoras podem ser efetivas na resolução de fístulas salivares. Conclusão: A identificação das estruturas glandulares afetadas é primordial para resolução do caso. A conduta deve ser individualizada para cada paciente tendo em conta a vasta gama de tratamentos disponíveis. Descritores: Côndilo Mandibular; Glândula Parótida; Complicações Pós-Operatórias.
\end{abstract}

\begin{abstract}
Introduction: The treatment of injuries to the facial skeleton sometimes requires extraoral approach for better visualization, reduction, and fixation of fractures. In condylar fractures the retromandibular approach is widely used in clinical practice, presenting predictability and adequate operative field, however it can generate complications such as paresthesia, sialocele, and parotid fistulas. Objective: This paper aims to report a clinical case of parotid fistula after retromandibular approach as well as the conduct applied in the treatment. Results: It is a consensus in the literature that acute injuries to the parotid gland should be promptly corrected, however there are many techniques to treat postoperative damages. Even extremely conservative management can be effective solve salivary fistulas. Conclusion: The identification of affected glandular structures is essential for case resolution. The conduct should be individualized for each patient taking into account the wide range of treatments available. Descriptors: Mandibular Condyle; Parotid Gland; Postoperative Complications.
\end{abstract}

\section{Resumen}

Introducción: El tratamiento de injurias al esqueleto facial requiere a veces acceso extra orales para una mejor visualización, reducción y fijación de las fracturas. En fracturas condilares el acceso retromandibular es ampliamente utilizado en la práctica clínica, presentando previsibilidad y campo quirúrgico adecuado, sin embargo puede generar complicaciones como parestesia, sialocele y fístulas parotídeas. Objetivo: relatar un caso clínico de fístula parotídea tras acceso retromandibular así como la conducta aplicada en el tratamiento. Resultados: Es consenso en la literatura que injurias agudas a la glándula parótida deben ser fácilmente corregidas, sin embargo daños postoperatorios crónicos presentan una amplia gama de tratamientos. Incluso conductas extremadamente conservadoras pueden ser efectivas en la resolución de fístulas salivares. Conclusión: La identificación de las estructuras glandulares afectadas es primordial para la resolución del caso. La conducta debe ser individualizada para cada paciente teniendo en cuenta la amplia gama de tratamientos disponibles.

Descriptores: Cóndilo Mandibular; Glándula Parótida; Complicaciones Posoperatorias.

\section{INTRODUÇÃO}

O tratamento de fraturas condilares dispõe de várias opções efetivas e bem documentadas na literatura. Seja em condutas conservadores ou abordagens cirúrgicas obtém-se taxas expressivas de sucesso. Em relação aos acessos cirúrgicos não há um consenso quanto ao melhor a ser utilizado, ficando a escolha do profissional, a critério da complexidade e localização da fratura, lacerações préexistentes, preferência do cirurgião, entre outros ${ }^{1-4}$.

$\mathrm{O}$ acesso retromandibular tem sido usado com sucesso para redução de fraturas mandibulares via extraoral, tendo como principais vantagens um campo de visualização adequado e uma angulação favorável para adaptação das placas e inserção dos parafusos. Dentre as possíveis complicações está a sialocele, que se caracteriza por acúmulo de saliva entre a pele e o tecido subcutâneo associado à lesão do parênquima glandular ou seu sistema de ductos. Quando este acúmulo encontra uma via de drenagem ocorre a formação de uma fístula salivar. Se proveniente do parênquima o tratamento conservador, em geral, soluciona a maioria dos casos, ao contrário de fístulas ductais que dificilmente tem uma resolução espontânea. O sucesso do tratamento depende de um diagnóstico precoce associado a conduta precisa. No acesso retromandibular é fundamental um correto fechamento do conjunto formado por músculo platisma, sistema músculo aponeurótico superficial e cápsula da glândula parótida diminuindo assim o risco de se formar uma sialocele ${ }^{5-9}$.
O propósito deste trabalho é descrever um caso clínico de fístula parotídea, desenvolvida após acesso retromandibular para redução de fratura condilar.

\section{CASO CLÍNICO}

Paciente do sexo masculino, 35 anos de idade, vítima de acidente ciclístico, apresentou fratura de côndilo mandibular esquerdo e parassínfise direita (Figura 1). Foi submetido a cirurgia para fixação interna das fraturas, sendo utilizado acesso retromandibular (Figura 2).

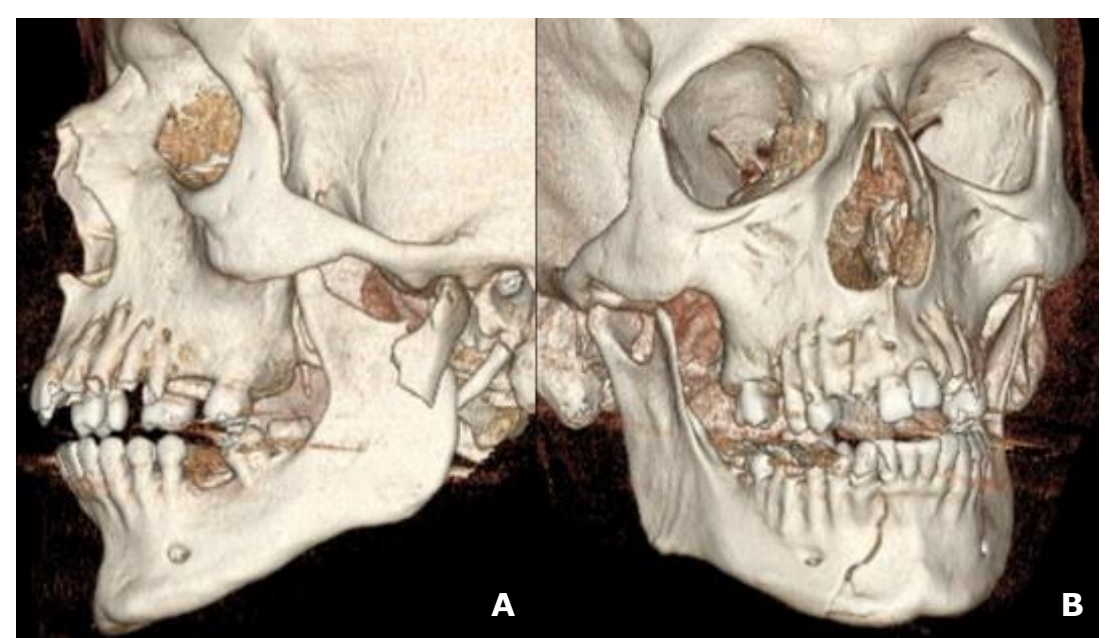

Figura 1: Fratura de côndilo mandibular esquerdo (A) e parassínfise mandibular direita (B). Fonte: acervo residência em cirurgia e traumatologia bucomaxilofacial HUOP, Cascavel, Paraná. 


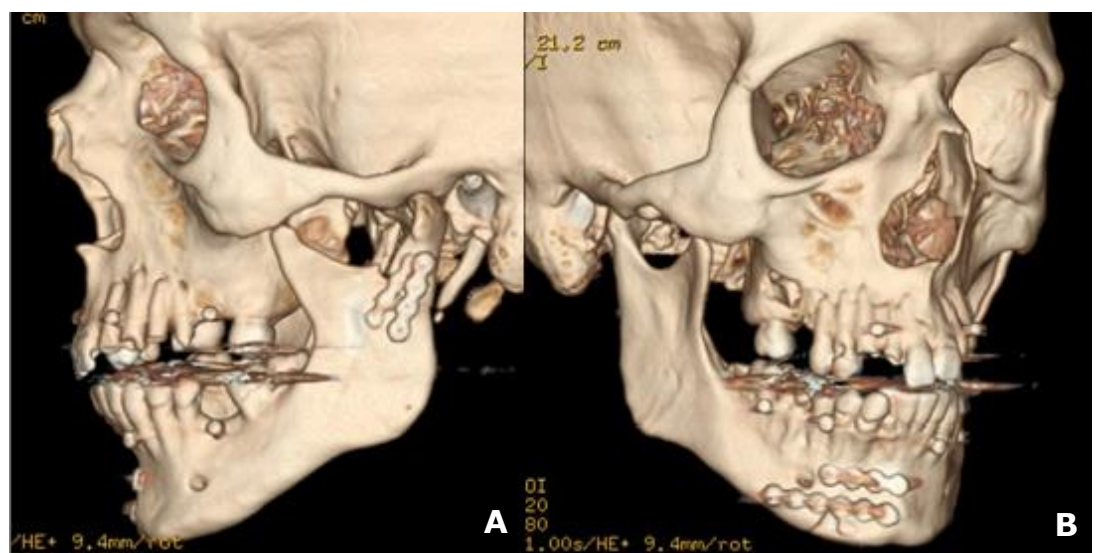

Figura 2: Fratura de côndilo mandibular esquerdo e parassínfise mandibular direita após fixação interna. Fonte: acervo residência em cirurgia e traumatologia bucomaxilofacial HUOP, Cascavel, Paraná.

Após 11 dias de pós-operatório apresentou aumento de volume flutuante a palpação em hemiface esquerda, paresia compatível com nervo marginal da mandíbula e sinais de infecção junto a extravasamento de líquido aquoso através da incisão extraoral (Figura 3). Ao estímulo intraoral apresentava pequena drenagem de saliva através da papila parotídea. Baseado nas características clínicas e abordagem cirúrgica realizada estabeleceu-se o diagnóstico de fístula parotídea. Não foi realizada punção do conteúdo devido ao comprometimento psicológico do paciente e às características infecciosas da área em questão.

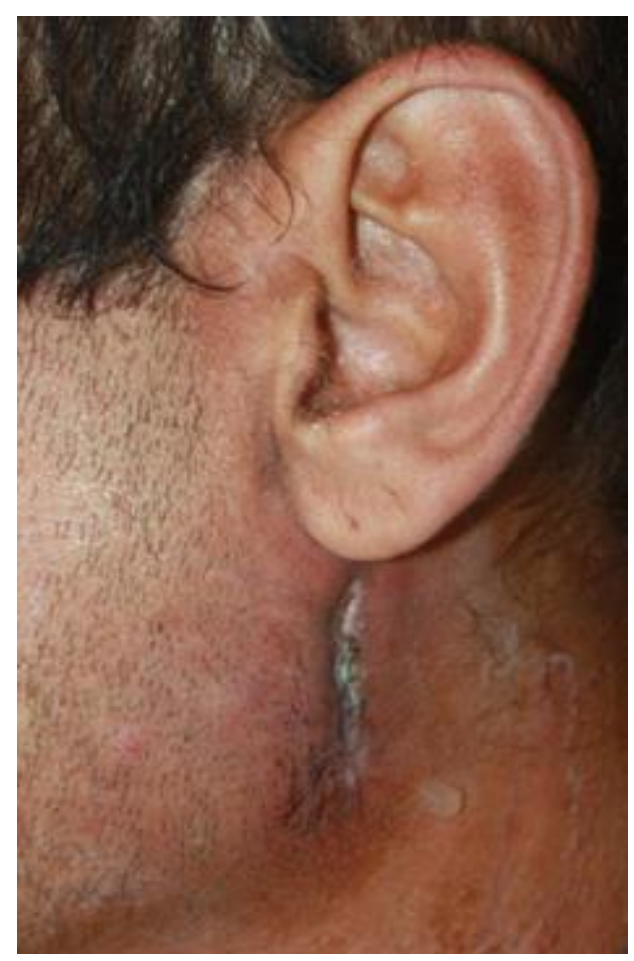

Figura 3: Pós-operatório 11 dias com sinais de infecção e drenagem de conteúdo através da incisão extraoral. Fonte: acervo residência em cirurgia e traumatologia bucomaxilofacial HUOP, Cascavel, Paraná.

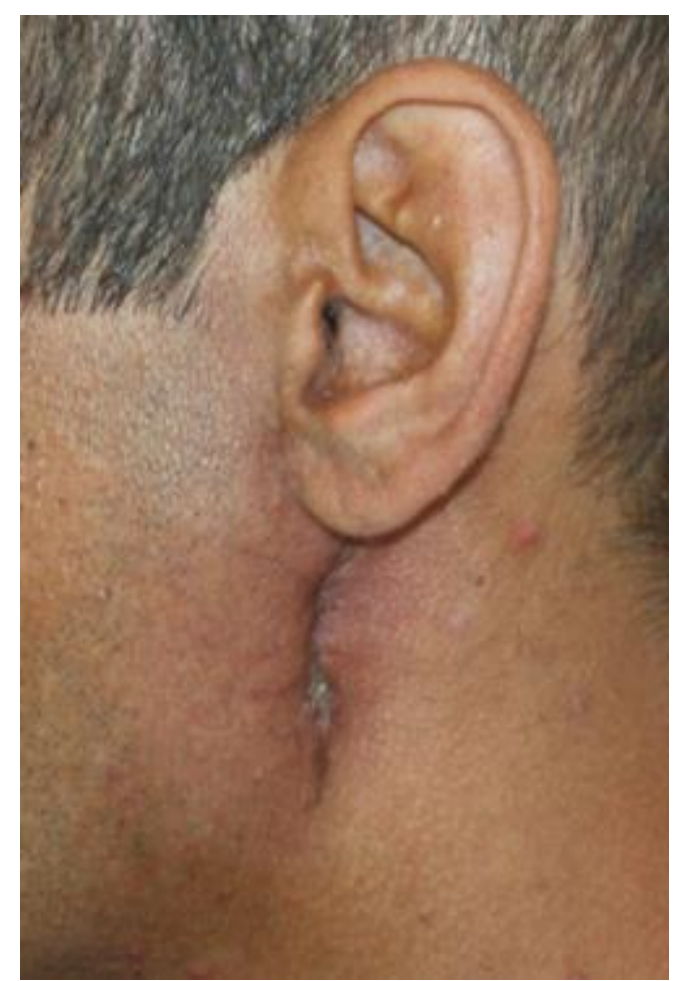

Figura 4: Pós-operatório de 32 dias com ausência de drenagem ou sinais infeciosos. Fonte: acervo residência em cirurgia e traumatologia bucomaxilofacial HUOP, Cascavel, Paraná.
O paciente foi medicado com cefalexina $500 \mathrm{mg}$ a cada 6 horas associada a metronidazol $500 \mathrm{mg}$ a cada 8 horas, ambos por 7 dias, e orientado a evitar alimentos cítricos na dieta. Após 18 dias apresentou redução da drenagem extraoral, porém não resolução do quadro infeccioso; foi instituído um novo ciclo de antibióticoterapia por mais 7 dias. Não foi realizado qualquer tipo de curativo compressivo durante o período. Após 25 dias apresentava drenagem discreta através da incisão extraoral e ausência de quaisquer sinais infeciosos. Foi mantida conduta conservadora, somente com orientação de dieta. Aos 32 dias de pós-operatório não havia qualquer tipo de drenagem via da incisão, ausência de sinais infeciosos e discreto aumento de volume (Figura 4). Completados 133 dias de acompanhamento o paciente foi liberado de alta, com a área da incisão sem qualquer sinal de recidiva ou alteração da normalidade (Figura 5).

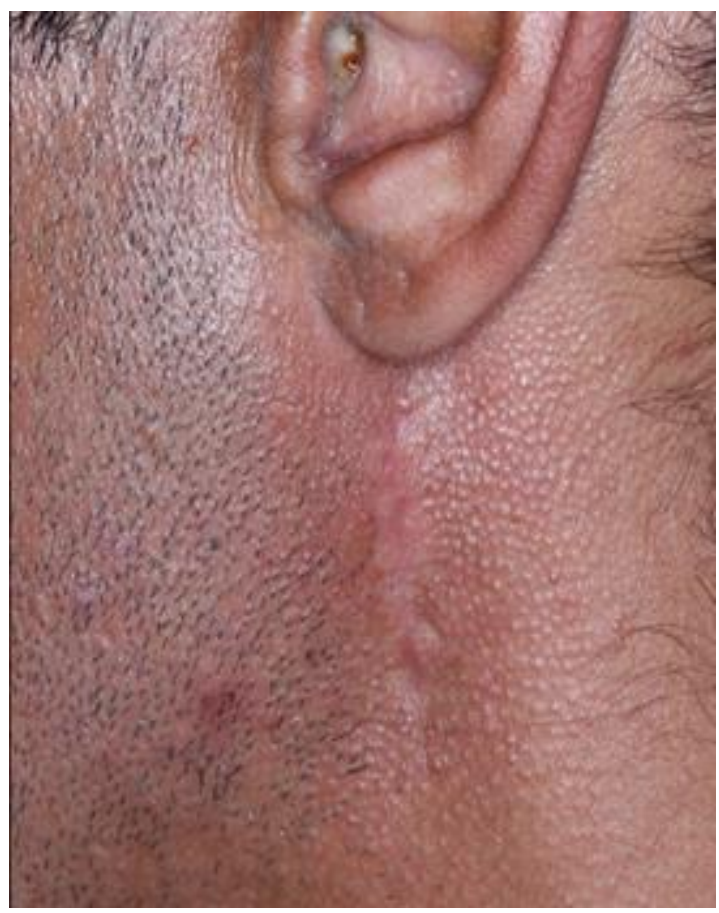

Figura 5: Pós-operatório de 133 dias sem qualquer sinal de recidiva. Fonte: acervo residência em cirurgia e traumatologia bucomaxilofacial HUOP, Cascavel, Paraná.

\section{DISCUSSÃO}

No que diz respeito aos tratamentos conservadores curativos compressivos são opção, porém com a realização de aspirações sucessivas do conteúdo não há como comprovar sua eficácia. Medicamentos anticolinérgicos não apresentam eficácia quando usados isoladamente e possuem o inconveniente de provocar náusea, êmese, xerostomia, taquicardia, retenção urinária, distúrbios de visão e insônia. A toxina botulínica atua paralisando a musculatura e afetando quimicamente a inervação da área, todavia exige um período prolongado de tratamento, com repetidas injeções, o que pode tornar o tratamento caro e pouco efetivo. A injeção de solução salina hipertônica figura como técnica efetiva, de baixo custo, biocompatível e com risco mínimo de lesão as estruturas

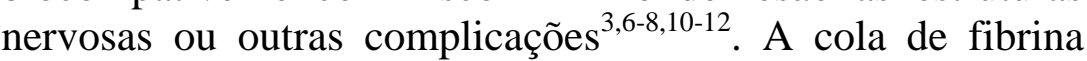
divide opiniões, comprovando sua efetividade nos estudos de Zwaveling et al. ${ }^{6}$, todavia sendo inativada pela saliva e resultando em recorrência da fístula nos trabalhos de Rajeev et al. ${ }^{8}$.

A intervenção cirúrgica em fístulas e sialoceles deve ser cuidadosamente avaliada. Recanalizar o trajeto afetado pode causar injúrias ao ducto parotídeo e danos ao nervo facial, principalmente em sialocele, onde seus limites são de difícil definição. A canalização é necessária por duas a três semanas até $\mathrm{o}$ ducto reestabelecer seu trajeto ${ }^{7,8}$.

No caso clínico apresentado, optou-se por realizar somente orientação de dieta, visto a drenagem regular de conteúdo, ausência de aumento de volume expressivo e função diminuída, porém presente da drenagem via ducto de Stensen. Em contraste com a literatura, observamos que a não intervenção relatada por nossa equipe necessitou de um 
período maior para atingir a resolução, com ausência da drenagem somente aos 32 dias de pós-operatório.

A diferenciação entre lesões ao parênquima e ao sistema de ductos parece ser fundamental para estabelecer o tratamento adequado, sendo a primeira passível de resolução espontânea e a segunda exigindo intervenção para possibilitar o reestabelecimento da drenagem e cicatrização de estruturas $\operatorname{afetadas}^{3,13}$.

Dentre os exames complementares disponíveis, a sialografia se mostra eficiente para diferenciar um simples acúmulo de um aumento de volume agravado pela estimulação da glândula. Nesta segunda situação pode se fazer necessário a canalização do conteúdo para possibilitar a cicatrização do parênquima glandular e uma correta drenagem. A ausência de drenagem intraoral através da papila é sinal importante para se atrelar a este exame $e^{3,13}$.

O diagnóstico deve ser individualizado levando em conta aspectos clínicos, histórico de trauma e abordagens cirúrgicas pelas quais o paciente passou. $\mathrm{O}$ presente relato de caso demostra que mesmo um tratamento conservador, se bem indicado, pode ser efetivo no tratamento de fístula salivar.

\section{CONCLUSÃO}

Com base nos dados apresentamos concluiu-se que:

$\checkmark$ Complicações como parestesia, paresia/paralisia, sialocele e fístulas salivares estão diretamente ligadas a técnica cirúrgica, sendo o profundo conhecimento desta fundamental para o sucesso na realização de acessos extra orais;

$\checkmark$ A identificação das estruturas glandulares afetadas é chave para se instituir uma conduta efetiva;

$\checkmark \mathrm{O}$ período de resolução mostra-se conduta dependente, exigindo o tratamento conservador apresentado do caso clínico um tempo maior se comparado a literatura;

$\checkmark$ A conduta deve ser individualizada para cada paciente tendo em conta a vasta gama de tratamentos disponíveis

\section{REFERENCIAS}

1. Ellis E 3rd, Dean J. Rigid fixation of mandibular condyle fractures. Oral Surg Oral Med Oral Pathol. 1993; 76(1):6-15.

2. Göçmen G, Varol A, Atalı O, Aktop S, Basa S. Retromandibular transparotid approach for subcondylar mandibular fractures: A retrospective study. Ulus Travma Acil Cerrahi Derg. 2017;23(3):251-7

3. Balaji SM. Parotid fistula from transparotid approach for mandibular subcondylar fracture reduction. Ann Maxillofac Surg. 2013;3(2):182-4

4. Ellis E 3rd, McFadden D, Simon P, Throckmorton G. Surgical complications with open treatment of mandibular condylar process fractures. J Oral Maxillofac Surg. 2000; 58(9):950-8

5. Dalla Torre D, Burtscher D, Widmann G, Pichler A, Rasse M, Puelacher W. Surgical treatment of mandibular condyle fractures using the retromandibular anterior transparotid approach and a triangular-positioned double miniplate osteosynthesis technique: A clinical and radiological evaluation of 124 fractures. J Craniomaxillofacial Surg. 2015; 43(6):944-9.

6. Zwaveling S, Steenvoorde P, da Costa SA. Treatment of postparotidectomy fistulae with fibrin glue. Acta Medica ((Hradec Kralove). 2006; 49(1):67-9

7. Chhabra N, Chhabra S, Kapila SA. Use of hypertonic saline in the management of parotid fistulae and sialocele: a report of 2 cases. J Maxillofac Oral Surg. 2009; 8(1):64-7
8. Rajeev R, Sajesh S, Jose M, Kumar ND. Sialocele: a rare sequlae of transparotid approach in subcondylar fracture management. Natl J Maxillofac Surg. 2016; 7(2):201-4.

9. Van Sickels JE. Management of parotid gland and duct injuries. Oral Maxillofac Surg Clin North Am. 2009; 21(2):243-6

10. Rao JK, Gehlot N, Laxmy V, Siwach V. Management of parotid fistula using hypertonic saline. Natl J Maxillofac Surg. 2011;2(2):177-80

11. Parekh D, Glezerson G, Stewart M, Esser J, Lawson HH. Post-traumatic parotid fistulae and sialoceles. A prospective study of conservative management in 51 cases. Ann Surg. 1989; 209(1):105-11.

12. Downie JJ, Devlin MF, Carton AT, Hislop WS. Prospective study of morbidity associated with open reduction and internal fixation of the fractured condyle by the transparotid approach. Br J Oral Maxillofac Surg. 2009;47(5):370-3

13. Gadodia A, Bhalla AS, Sharma R, Thakar A, Parshad R. MR Sialography of iatrogenic sialocele: Comparison with conventional sialography. Dentomaxillofac Radiol. 2011; 40(3):147-53

\section{CONFLITO DE INTERESSES}

Os autores declaram não haver conflitos de interesse.

\section{AUTOR PARA CORRESPONDÊNCIA}

\section{Felipe Aurélio Guerra}

felipeaurelioguerra@gmail.com

Submetido em 18/02/2018

Aceito em 19/03/2018 